\title{
$\mathrm{KJCCM}$
}

Korean J Crit Care Med

2017 November 32(4):356-358 / https://doi.org/10.4266/kjccm.2017.00556

ISSN 2383-4870 (Print) · ISSN 2383-4889 (Online)

Editorial

\section{Lung Ultrasound in the Critically Ill}

\section{Jin Sun Cho}

Department of Anesthesiology and Pain Medicine, Yonsei University College of Medicine, Seoul, Korea

Until recently, the lung was considered "forbidden territory" for ultrasound. With lung ultrasound, however, the amount of lung consolidation and pleural effusion can be assessed semiquantitatively. Lung ultrasound consists of the identification of 10 signs, and there are several well-established protocols such as the BLUE (Bedside Lung Ultrasonography in Emergency) protocol for diagnosing acute respiratory failure and the FALLS (Fluid Administration Limited by Lung Sonography) protocol for managing acute circulatory failure. The BLUE protocol is a fast protocol that defines eight profiles, correlated with six diseases seen in $97 \%$ of patients admitted to the intensive care unit (ICU). With this protocol, it becomes possible to differentiate between pulmonary edema, pulmonary embolism, pneumonia, chronic obstructive pulmonary disease, asthma, and pneumothorax [1]. The FALLS protocol uses the potential of lung ultrasound for the early demonstration of fluid overload at an infra-clinical level [2]. It is used in patients with acute respiratory failure, allowing a sequential search for obstructive, cardiogenic, hypovolemic, and distributive shock using simple real-time echocardiography in combination with lung ultrasound, with the appearance of B lines considered to be the endpoint of fluid therapy. In addition, ultrasound can help to guide airway management in a patient with acute respiratory distress who needs to be intubated and mechanically ventilated (PINK protocol). In a patient with acute respiratory distress who is often ventilated and difficult to transport, computed tomography (CT) is not an easy option, and lung ultrasound can help to predict difficult airway and proper endotracheal tube size, or to confirm proper endotracheal tube placement with avoidance of desaturation during CT [3]. In addition, lung ultrasound can be used to determine the cause of fever distinguishing pneumonia from atelectasis [4], and to rule out pneumothorax, hypovolemia, pulmonary embolism and pericardial tamponade in cardiac arrest (SESAME protocol) [5].

In the critical care setting, lung ultrasound is increasingly used, as it allows bedside visualization of the lungs. Critical care ultrasound is a combination of simple protocols, with lung ultrasound being a basic application, allowing the assessment

\section{Jin Sun Cho \\ Department of Anesthesiology and Pain Medicine, Yonsei University College of Medicine, 50-1 Yonsei-ro, Seodaemun- gu, Seoul 03722, Korea \\ Tel: +82-2-2224-1286 \\ Fax: +82-2-2227-6517 \\ E-mail: chis0214@yuhs.ac \\ ORCID \\ Jin Sun Cho \\ http://orcid.org/0000-0002-5408-4188 \\ *No potential conflict of interest relevant to this article was reported}

cc This is an Open Access article distributed under the terms of the Creative Commons Attribution Non-Commercial License (http://creativecommons.org/ licenses/by-nc/4.0/) which permits unrestricted non-commercial use, distribution, and reproduction in any medium, provided the original work is properly cited. Copyright (c) 2017 The Korean Society of Critical Care Medicine 
of urgent diagnoses and therapeutic decisions. Although chest radiographs (CXR) and CT are mostly used for daily or prompt evaluation of lung in the ICU, there are significant drawbacks such as the huge radiation hazard, need for transportation, and risk of contrast use. On the other hand, lung ultrasound has advantages of absence of radiation, bedside availability, good reproducibility, and cost efficiency [6]. Lung ultrasound has more accuracy than bedside CXR and roughly the same accuracy as CT. Ultrasound is far superior for the detection of pneumothorax and pleural effusion compared with CXR and provides accurate quantitative data regarding the volume of pleural effusions, lung consolidations, and pneumotho$\operatorname{rax}[7,8]$. Although supine portable CXR is notoriously unreliable in the evaluation of pneumothorax, in the absence of tube thoracostomy or subcutaneous emphysema, ultrasound has sensitivity and specificity superior to CXR for the detection of pneumothorax [9]. Plain CXR is most sensitive for pleural effusion when the patient is in the upright or lateral decubitus position, but optimal positioning is difficult in the ICU. Ultrasound can detect pleural effusions with a sensitivity and specificity of $93 \%$ when CT is used as a gold standard [10].

In an article of the Korean Journal of Critical Care Medicine, Kang et al. [11] reported the usefulness of lung ultrasound to detect pulmonary complication including pulmonary edema and pneumonia especially in the surgical ICUs, where pulmonary complications are major causes of morbidity and mortality. While previous studies on lung ultrasound were mostly for use in medical ICUs, the authors demonstrated the usefulness of lung ultrasound in the surgical ICU. Their indications for lung ultrasound included hypoxemia, abnormal CXR without hypoxemia, fever, and difficult weaning. Lung ultrasound was helpful for diagnosis of pneumonia, atelectasis, pulmonary edema, or a combination of these diseases. In addition, lung ultrasound detected lung parenchymal consolidation with air bronchogram, pulmonary edema, and pneumonia even in patients without CXR abnormalities. In the surgically ill and injured patients, combined with venous, cardiac, and abdominal examination, ultrasound investigation of lung can provide an overview of cardiac performance and intravascular volume and practically guide management for hemodynamic optimization.

Lung ultrasound can be extended from neonates to adults, and from medical to surgical and several other disciplines (anesthesiology, emergency medicine, etc.). The higher rate of detection of ultrasound, combined with its ease and increasing accessibility, makes for a powerful diagnosis in the ICU. Although lung ultrasound requires acquisition of an ultrasound machine and training of physicians, it allows a critical care provider to quickly respond to a majority of critical situations. Therefore, lung ultrasound could be a reasonable, fully operational, bedside gold standard in the ICU.

\section{References}

1. Lichtenstein DA, Mezière GA. Relevance of lung ultrasound in the diagnosis of acute respiratory failure: the BLUE protocol. Chest 2008;134:117-25.

2. Lichtenstein D. Fluid administration limited by lung sonography: the place of lung ultrasound in assessment of acute circulatory failure (the FALLS-protocol). Expert Rev Respir Med 2012;6:155-62.

3. Terkawi AS, Karakitsos D, Elbarbary M, Blaivas M, Durieux ME. Ultrasound for the anesthesiologists: present and future. ScientificWorldJournal 2013;2013:683685.

4. Lichtenstein D, Mezière G, Seitz J. The dynamic air bronchogram: a lung ultrasound sign of alveolar consolidation ruling out atelectasis. Chest 2009;135:1421-5.

5. Lichtenstein DA. How can the use of lung ultrasound in cardiac arrest make ultrasound a holistic discipline: the example of the SESAME-protocol. Med Ultrason 2014;16:252-5.

6. Lichtenstein DA, Mezière GA, Lagoueyte JF, Biderman P, Goldstein I, Gepner A. A-lines and B-lines: lung ultrasound as a bedside tool for predicting pulmonary artery occlusion pressure in the critically ill. 
Chest 2009;136:1014-20.

7. Lichtenstein D, Mezière G, Biderman P, Gepner A. The "lung point": an ultrasound sign specific to pneumothorax. Intensive Care Med 2000;26:1434-40.

8. Oveland NP, Lossius HM, Wemmelund K, Stokkeland PJ, Knudsen L, Sloth E. Using thoracic ultrasonography to accurately assess pneumothorax progression during positive pressure ventilation: a comparison with CT scanning. Chest 2013;143:41522.

9. Blaivas M, Lyon M, Duggal S. A prospective com- parison of supine chest radiography and bedside ultrasound for the diagnosis of traumatic pneumothorax. Acad Emerg Med 2005;12:844-9.

10. Lichtenstein DA. Ultrasound in the management of thoracic disease. Crit Care Med 2007;35(5 Suppl): S250-61.

11. Kang HK, So HJ, Kim DH, Koo HK, Park HK, Lee SS, et al. The use of lung ultrasound in a surgical intensive care unit. Korean J Crit Care Med 2017;32:323-32. 